\title{
AMALGAMATION AND INVERSE AND REGULAR SEMIGROUPS
}

BY

\author{
T. E. HALL
}

\begin{abstract}
A method for proving the embeddability of semigroup amalgams is introduced. After providing necessary and sufficient conditions in terms of representations for the weak embeddability of a semigroup amalgam, it successfully deals with the embedding of inverse semigroup amalgams into inverse semigroups and the embedding of an amalgam of regular semigroups whose core is full in each member.
\end{abstract}

Most of the known results on amalgamation of semigroups, almost all of which are due to J. M. Howie, have now been proved by a method introduced in [7] involving a countably infinite number of steps, each step extending a representation of a semigroup [7], [8], [14], [15]; the main results thus proved concern amalgamation over a common unitary subsemigroup, an almost unitary subsemigroup, an inverse subsemigroup, a two-element subsemigroup, and the embedding of an inverse semigroup amalgam in an inverse semigroup. We give here a method of proof that avoids this infinite number of steps.

First it yields a necessary and sufficient condition, in terms of representations, for the weak embeddability of a semigroup amalgam (defined below).

This gives then a short proof of [7, Theorem 8] (see also Howie's text [9] for an exposition), namely that inverse semigroups have the strong amalgamation property. Further we are able to show that finiteness can be preserved in the embedding of an amalgam $\left(S_{i}, i \in I ; U\right)$ of inverse semigroups if the common inverse subsemigroup $U$ is full in each $S_{i}$, i.e. contains all the idempotents of each $S_{i}$; in general, finiteness cannot be preserved [7, §3].

Further we are able to show that, in fact, regular semigroups can be amalgamated over a full regular subsemigroup, also with finiteness being preserved. In general, an amalgam of regular semigroups (even left regular bands) cannot be weakly embedded in a semigroup [8, Remark 7]. In the final section we consider semigroups that are unions of groups.

Received by the editors August 9, 1977.

AMS (MOS) subject classifications (1970). Primary $20 \mathrm{M} 10$.

Key words and phrases. Amalgamation, inverse semigroups, regular semigroups.

(c) American Mathematical Society 1979 


\section{Preliminaries.}

ReSULT 1 [4, RESULT 9]. If $U$ is a regular subsemigroup of a semigroup $S$ then Green's relation $\mathcal{L}$ on $U$ is the restriction to $U$ of $\mathcal{L}$ on $S$.

ResUlt 2 [5, TheOREM 14]. If $E$ is any set of idempotents of a semigroup $S$ such that there is a regular subsemigroup $U$ of $S$ with $E$ as the set of all idempotents of $U$, then there is a unique maximum regular subsemigroup of $S$ with $E$ as its set of idempotents.

For any set $X$ we denote by $\mathscr{P} \mathscr{T}(X)$ and by $\mathscr{T}(X)$ the semigroups of all partial and all total transformations of $X$, respectively, under composition of binary relations. For any set $X$ and any semigroup $S$ we call a homomorphism $\rho$ of $S$ into $\mathscr{P} \mathcal{T}(X)$ a representation of $S$ by partial transformations of $X$. By an $S$-orbit of $\rho$ or a $\rho$-orbit we mean any minimal (with respect to the partial ordering $\subseteq$ ) nonempty subset of $X$ closed under the taking of images and pre-images by the functions $\rho_{s}, s \in S$. The $\rho$-orbits thus include the singleton sets $\{x\}$ for each $x \in X$ which is not in the domain or range of any $\rho_{s}, s \in S$. We note that the action of $S$ on a $\rho$-orbit is not necessarily transitive, but that, of course, $X$ is the disjoint union of its $\rho$-orbits. We call the partial unary algebra $\left(X,\left\{\rho_{s}: s \in S\right\}\right)$, consisting of $X$ and an indexed set of partial unary operations indexed by elements from $S$, an $S$-system, and of course, two $S$-systems $\left(X,\left\{\rho_{s}: s \in S\right\}\right)$ and $\left(X^{\prime},\left\{\rho_{s}^{\prime}: s \in S\right\}\right)$ are called isomorphic or equivalent if there is a bijection $\phi: X \rightarrow X^{\prime}$ such that $\phi^{-1} \rho_{s} \phi=$ $\rho_{s}^{\prime}$ for all $s \in S$. As is usual for all structures, $\left(X,\left\{\rho_{s}: s \in S\right\}\right)$ is often denoted by just $X$, the context telling us the partial operations $\rho_{s}, s \in S$. For a subset $X^{\prime}$ of $X$ we denote by $\rho \| X^{\prime}$ the function from $S$ into $\mathcal{P} \mathcal{T}(X)$ which maps each $s \in S$ to $\rho_{s} \mid X^{\prime}$, the partial transformation of $X$ obtained by restricting the domain of $\rho_{s}$ to $X^{\prime} ; \rho \| X^{\prime}$ is a representation if $X^{\prime} \rho_{s} \subseteq X^{\prime}$ for all $s \in S$.

By a class $\mathcal{C}$ of representations of a class $\mathcal{Q}$ of semigroups we simply mean a class each object of which is a representation of some semigroup from $\mathbb{Q}$; three such classes consist of all representations, and the representations by total and by one-to-one partial transformations.

Let $U$ and $S$ be any semigroups such that $U \leqslant S$ and let $\mathcal{C}$ be a class of representations of $\{U, S\}$. We say that $U$ has the [orbit preserving] representation extension property in $S$ for the class $\mathcal{C}$ if for any representation $\rho$ : $U \rightarrow \mathscr{P} \mathcal{T}(X)$ from $\mathcal{C}$ there is a set $Y$ disjoint from $X$ and a representation $\alpha$ : $S \rightarrow \mathcal{P} \mathcal{F}(X \cup Y)$ from $\mathcal{C}$ such that $\alpha_{u} \mid X=\rho_{u}$ [and further $Y \alpha_{u} \subseteq Y$ ] for all $u \in U$. We permit ourselves to omit the phrase "for the class $\mathcal{C}$ " when $\mathcal{C}$ is the class of all representations.

Using the standard method of embedding $\mathscr{P} \mathcal{T}(X)$ into $\mathcal{T}(X \cup 0)$, where $0 \notin X[1, \S 11.1]$, we can easily see that $U$ has the [orbit preserving] representation extension property in $S$ for the class of representations by total transformations if $U$ has this property in $S$ for the class of all representations. 
ResUlt 3 [8, TheOREM 1]. If $U$ is an inverse subsemigroup of a semigroup $S$, then $U$ has the representation extension property in $S$ for the class of representations by total transformations.

Let $\mathcal{Q}$ be any class of algebras. If, for some index set $I,\left\{S_{i}: i \in I\right\}$ is an indexed set of algebras from $\mathcal{Q}$ having a common subalgebra $U$ also in $\mathcal{Q}$, then the list $\left(S_{i}, i \in I ; U\right)$ is called an amalgam from $\mathbb{Q}$. If there exist an algebra $W$ and monomorphisms $\phi_{i}: S_{i} \rightarrow W, i \in I$, agreeing on $U$ (i.e. $\phi_{i}\left|U=\phi_{j}\right| U$ for all $\left.i, j \in I\right)$ then we say the amalgam $\left(S_{i}, i \in I ; U\right)$ is weakly [strongly] embeddable in $W$ [if also $\left(S_{i} \phi_{i}\right) \cap\left(S_{j} \phi_{j}\right)=U \phi_{i}$ for all distinct $i$, $j \in I$ ]. An algebra $U \in \mathbb{Q}$ is called a weak [strong] amalgamation base in $\mathbb{Q}$ if every amalgam from $\mathcal{Q}$ of the form $\left(S_{i}, i \in I ; U\right)$ is weakly [strongly] embeddable in an algebra from $\mathcal{Q}$. If an amalgam of the form $(S, S ; U)$ is strongly embeddable in an algebra from $\mathbb{Q}$ we say $U$ is closed in $S$ (within $\mathscr{Q}$ ). If $U$ is closed in $S$ within $\mathbb{Q}$ for all $U, S \in \mathbb{Q}$ with $U<S$, then we say $\mathbb{Q}$ has the special amalgamation property. If every amalgam from $\mathbb{Q}$ is weakly [strongly] embeddable in an algebra from $\mathcal{Q}$ then we say $\mathcal{Q}$ has the weak [strong] amalgamation property.

RESULT 4 [9, THEOREM 4.11]. The class of inverse semigroups has the special amalgamation property.

\section{A necessary and sufficient condition.}

THEOREM 1. An amalgam $\left(S_{i}, i \in I ; U\right)$ of semigroups is weakly embeddable in a semigroup if and only if, for all $i \in I$, there is a set $Z_{i}$ and a faithful representation $\alpha^{(i)}: S_{i} \rightarrow \mathcal{P} \mathcal{T}\left(Z_{i}\right)$ such that the representations $\alpha^{(i)} \mid U, i \in I$, of $U$ contain precisely the same isomorphism types of $U$-orbits (i.e. for all $i, j \in I$, for each $U$-orbit of $\alpha^{(i)} \mid U$ there is an isomorphic $U$-orbit of $\left.\alpha^{(j)} \mid U\right)$.

Proof. (i) Suppose such representations exist. We consider first the case $I=\{1,2\}$, say. Put $S=S_{1}, T=S_{2}, \alpha=\alpha^{(1)}$ and $\beta=\alpha^{(2)}$ and let $\lambda$ be any infinite cardinal such that $\lambda \geqslant\left|Z_{1}\right|,\left|Z_{2}\right|$. Let $\alpha^{\prime}: S \rightarrow \mathcal{P} \mathscr{T}\left(Z_{1}^{\prime}\right)$ be the sum of $\lambda$ copies of $\alpha: S \rightarrow \mathcal{P} \mathscr{T}\left(Z_{1}\right)$ and let $\beta^{\prime}: T \rightarrow \mathscr{P} \mathscr{T}\left(Z_{2}^{\prime}\right)$ be the sum of $\lambda$ copies of $\beta: T \rightarrow \mathscr{P} \mathcal{T}\left(Z_{2}\right)$.

Now $\alpha^{\prime} \mid U$ and $\beta^{\prime} \mid U$ each have exactly $\lambda$ copies of each isomorphism type of $U$-orbit that either of them contains, so they are equivalent; i.e. there is a bijection $\phi: Z_{1}^{\prime} \rightarrow Z_{2}^{\prime}$ such that $\phi^{-1} \alpha_{u}^{\prime} \phi=\beta_{u}^{\prime}$ for all $u \in U$.

We now define a representation $\alpha^{\prime \prime}: S \rightarrow \mathcal{P} \mathcal{T}\left(Z_{2}^{\prime}\right)$ by $\alpha_{s}^{\prime \prime}=\phi^{-1} \alpha_{s}^{\prime} \phi$ for all $s \in S$. Then $\alpha^{\prime \prime}\left|U=\beta^{\prime}\right| U$ and $\alpha^{\prime \prime}$ and $\beta^{\prime}$ are faithful on $S$ and $T$, respectively, so $(S, T ; U)$ is weakly embeddable. We may similarly deal with the arbitrary case by taking $\lambda \geqslant\left|Z_{i}\right|$ for all $i \in I$ and by taking $\lambda$ copies of each $\alpha^{(i)}$.

(ii) Suppose $\left(S_{i}, i \in I ; U\right)$ is weakly embedded in a semigroup $W$ by monomorphisms $\phi_{i}: S_{i} \rightarrow W$ agreeing on $U$. Let $\rho: W \rightarrow \mathscr{P} \mathcal{T}\left(W^{l}\right)$ be the 
extended right regular representation of $W$. Put $Z_{i}=W^{1}$ and $\alpha^{(i)}=\phi_{i} \rho$ for all $i \in I$. Then, in fact, the representations $\alpha^{(i)} \mid U$ are all identical. We also see that the theorem remains valid if $\mathscr{P} \mathcal{T}\left(Z_{i}\right)$ is replaced by $\mathcal{T}\left(Z_{i}\right)$.

THEOREM 2. If $U$ is any subsemigroup of any semigroup $S$ such that $U$ has the orbit preserving representation extension property in $S$, then for any infinite cardinal $\kappa \geqslant|S|$, there is a set $Z$ and a faithful representation $\alpha: S \rightarrow \mathscr{P} \mathcal{T}(Z)$ such that the orbits of $\alpha \mid U$, up to isomorphism, are precisely all the possible $U$-orbits on sets of size at most $\kappa$.

Proof. For any semigroup $U$ and any cardinal $\kappa$ let $\left\{X_{j}: j \in J\right\}$ be an indexed set of sets, pairwise disjoint, and for each $j \in J$ let $\rho^{(j)}: U \rightarrow \mathscr{P} \mathcal{T}\left(X_{j}\right)$ be a representation of $U$, such that each $X_{j}$ is a $\rho^{(j)}$-orbit, but further such that for any arbitrary $U$-orbit $O$ of size at most $\kappa$, there is precisely one $j \in J$ such that $X_{j}$ and $O$ are isomorphic $U$-systems. (If $\kappa$ is infinite then $|J|<2^{\kappa}$.)

Put $X=\cup_{j \in J} X_{j}$ and let $\rho: U \rightarrow \mathcal{P} \mathcal{T}(X)$ be the sum of the representations $\rho^{(j)}: U \rightarrow \mathscr{P} \mathcal{G}\left(X_{j}\right), j \in J$ (i.e. we define $\rho$ by $\rho_{u}=\bigcup_{j \in J} \rho_{u}^{(j)}$ for each $u \in U$ ).

Now let $S$ be any semigroup with $U$ as a subsemigroup and such that $U$ has the representation extension property in $S$. Then for each $j \in J$ there exist a set $Y_{j}$ disjoint from $X_{j}$ and a representation $\alpha^{(j)}: S \rightarrow \mathscr{P} \mathcal{T}\left(X_{j} \cup Y_{j}\right)$ such that $\alpha_{u}^{(j)} \mid X_{j}=\rho_{u}^{(j)}$ for all $u \in U$. Without loss of generality we can assume that $X_{j} \cup Y_{j}=X_{j} \cup\left(\cup_{s \in S} X_{j} \alpha_{s}\right)$ and that for all distinct $j, k \in J, X_{j}, X_{k}, Y_{j}, Y_{k}$ are pairwise disjoint. Put $Y=\cup_{j \in J} Y_{j}$ and let $\alpha: S \rightarrow \mathcal{P} \mathcal{T}(X \cup Y)$ be the sum of the representations $\alpha^{(j)}, j \in J$. Let $O$ be any $U$-orbit of $\alpha \mid U$. Then for some $j \in J, O \subseteq X_{j} \cup Y_{j}$ whence

$$
|O| \leqslant\left|X_{j} \cup Y_{j}\right| \leqslant\left|X_{j}\right|+\left|X_{j} \times S\right|=\left|X_{j}\right|+\left|X_{j}\right||S| .
$$

Thus if $\kappa$ is an infinite cardinal such that $\kappa \geqslant|S|$, then $O$ is also a $U$-orbit with size at most $\kappa$.

If $U$ has the orbit preserving extension property in $S$ then we can assume that further, for all $j \in J$, for all $u \in U, Y_{j} \alpha_{u}^{(j)} \subseteq Y_{j}$, i.e. that $X_{j}$ is a $U$-orbit of $\alpha \mid U$. To make $\alpha$ faithful on $S$ we need merely take the sum of $\alpha$ and the extended right regular representation of $S$. This completes the proof.

Corollary 3 [8, Theorem 27(i)]. An amalgam $\left(S_{i}, i \in I ; U\right)$ of semigroups is weakly embeddable if $U$ has the orbit preserving representation extension property in each $S_{i}$ for the class of representations by total transformations.

Proof. The proof of Theorem 2 shows that Theorem 2 is still valid if we consider only representations by total transformations. The corollary follows immediately from Theorem 1 and this modified version of Theorem 2. Note that by [8, Theorem $27(\mathrm{i})]$ the amalgam $\left(S_{i}, i \in I ; U\right)$ is actually strongly embeddable. 
THeOREM 4 [7, THEOREM 5]. Let $U$ be any inverse subsemigroup of any inverse semigroup $S$. Then $U$ has the orbit preserving representation extension property in $S$ for the class of representations by one-to-one partial transformations.

Proof. Take any set $X$ and any representation $\rho: U \rightarrow G(X)$, the semigroup of one-to-one partial transformations of $X$. Using the standard method of [1, $\S 11.1$ ], we take an element, 0 say, not in $X$, and for each $u \in U$ define a total transformation $\gamma_{u}$ of $X \cup 0=X \cup\{0\}$ by $\gamma_{u}=\rho_{u} \cup(X \times\{0\}) \cup\{(0,0)\}$. Then the map $\gamma: U \rightarrow \mathscr{T}\{X \cup 0\}, u \mapsto \gamma_{u}$ is easily seen to be a homomorphism. By Result 3 there is a set $Y$ disjoint from $X \cup 0$ and a representation $\alpha: S \rightarrow \mathscr{T}(X \cup 0 \cup Y)$ such that $\alpha_{u} \mid(X \cup 0)=\gamma_{u}$ for all $u \in$ $U$.

As in [12] or [13, Theorem 1], we define a representation $\alpha^{*}: S \rightarrow g(X \cup 0$ $\cup Y)$ by $\alpha_{s}^{*}=\alpha_{s} \mid\left((X \cup 0 \cup Y) \alpha_{s^{-1}}\right)$ for all $s \in S$. Easy checking shows that $\alpha_{u}^{*} \mid X=\rho_{u}$ for all $u \in U$. Further, $(Y \cup 0) \alpha_{u}^{*} \subseteq Y \cup 0$ for all $u \in U$, since for any $y \in Y \cup 0$ such that $y \in$ Domain $\alpha_{u}^{*}$, we have $\left(y \alpha_{u}^{*}\right) \alpha_{u-1}^{*}=y \in Y \cup$ 0 so $y \alpha_{u}^{*} \notin X$. This completes the proof of the theorem.

THEOREM 5 [7, THEOREM 8]. The class of inverse semigroups has the strong amalgamation property.

Proof. Take any amalgam $(S, T ; U)$ of inverse semigroups. Using Theorem 4 we may modify the proof of Theorem 2 to show that there is a set $Z$ and a faithful representation $\alpha: S \rightarrow \mathscr{G}(Z)$ such that $\alpha \mid U$ contains, up to isomorphism, precisely the $U$-orbits of size at most $\kappa$ in the class of representations by one-to-one partial transformations. Without modification the proof of Theorem 1 yields faithful representations of $S$ and $T$ in $g\left(Z_{2}^{\prime}\right)$, agreeing on $U$. Thus the amalgam is weakly embeddable in an inverse semigroup $W$, say by monomorphisms $\phi: S \rightarrow W, \psi: T \rightarrow W$ agreeing on $U$. By Result 4 there exist an inverse semigroup $W^{\prime}$ and monomorphisms $\phi_{1}$ : $W \rightarrow W^{\prime}, \phi_{2}: W \rightarrow W^{\prime}$ agreeing precisely on $U \phi=U \psi$. Then the monomorphisms $\phi \phi_{1}: S \rightarrow W^{\prime}$ and $\psi \phi_{2}: T \rightarrow W^{\prime}$ agree precisely on $U$, giving that the amalgam $(S, T ; U)$ is strongly embeddable in an inverse semigroup. An easy transfinite induction argument gives the strong embeddability of arbitrary amalgams of inverse semigroups.

3. Amalgamation over a full regular subsemigroup. Using Lallement's reformulation [10, Proposition 2.2] of Preston's representation result [11, Theorem 2], for any semigroup $S$ we define for each element $s \in S$, a partial transformation $\rho(S)_{s}$ of $S$ by

$$
\rho(S)_{s}=\{(x, x s) \in S \times S: x \Re x s \text { in } S\},
$$

and we define $\rho(S): S \rightarrow \mathcal{P} \mathcal{T}(S)$ to be the function mapping each $s \in S$ to 
$\rho(S)_{s}$. Then $\rho(S)$ is a representation of $S$, and is just the Preston-Vagner representation (as in $[1, \S 1.9])$ when $S$ is an inverse semigroup.

Dually, we define $\lambda(S): S \rightarrow \mathscr{P} \mathcal{T}(S)$ by

$$
\lambda(S)_{s}=\{(x, s x) \in S \times S: x \in s x \text { in } S\}
$$

for each $s \in S$. Then $\lambda(S): S \rightarrow \mathscr{P} \mathcal{F}(S)^{*}$, the semigroup dual to $\mathscr{P} \mathcal{F}(S)$, is a homomorphism.

If $S$ is regular then the map

$$
(\lambda(S), \rho(S)): S \rightarrow \mathcal{P} \mathcal{T}(S)^{*} \times \mathcal{P} \mathcal{T}(S), \quad s \mapsto\left(\lambda(S)_{s}, \rho(S)_{s}\right),
$$

is a monomorphism [10, Proposition 2.2].

LEMMA 6. If $U$ is a full regular subsemigroup of a regular semigroup $S$, then the amalgam $(S, S ; U)$ is strongly embeddable in a semigroup (i.e. $U$ is closed in $S$ ). If $S$ is finite then the amalgam is strongly embeddable in a finite semigroup.

Proof. For each $u \in U$ it is clear that $U \rho(S)_{u} \subseteq U$; we show that also $(S \backslash U) \rho(S)_{u} \subseteq S \backslash U$. Suppose to the contrary that there is an element $b \in S \backslash U$ such that $b \rho(S)_{u} \in U$. Let $b^{\prime}$ be any inverse of $b$ in $S$. Then $b u$, $b^{\prime} b,\left(b^{\prime} b\right) u \in U$ and $b \Re b u$ in $S$ so $b^{\prime} b \Re b^{\prime} b u$ in $S$ and hence in $U$ (Result 1). Thus there exists an element $v \in U^{1}$ such that $b^{\prime} b=b^{\prime} b u v$ whence $b=b b^{\prime} b=b b^{\prime} b u v=(b u) v \in U$, a contradiction, as required.

Let $S^{\prime}$ be a set disjoint from $S$ and of the same cardinality, and let $S \rightarrow S^{\prime}$, $x \mapsto x^{\prime}$ be a bijection. Make $S^{\prime}$ a copy of the $S$-system $S$ by defining $\rho^{\prime}(S)$ : $S \rightarrow \mathscr{P} \mathcal{T}\left(S^{\prime}\right)$ by $x^{\prime} \rho^{\prime}(S)_{s}=(x s)^{\prime}$ for all $x^{\prime} \in S^{\prime}, s \in S$ such that $x \in$ Domain $\rho(S)_{s}$.

Define a permutation $\phi: S \cup S^{\prime} \rightarrow S \cup S^{\prime}$ by, for all $a \in U, b \in S \backslash U$, $a \phi=a, a^{\prime} \phi=a^{\prime}, b \phi=b^{\prime}, b^{\prime} \phi=b$. Let $\alpha: S \rightarrow \mathcal{P} \mathcal{T}\left(S \cup S^{\prime}\right)$ be the sum of $\rho(S)$ and $\rho^{\prime}(S)$ and define $\beta: S \rightarrow \mathcal{P} \mathcal{T}\left(S \cup S^{\prime}\right)$ by $\beta_{s}=\phi^{-1} \alpha_{s} \phi$ for all $s \in S$. It is easy to see that $\alpha_{u}=\beta_{u}$ for all $u \in U$. Further, for any $s \in S \backslash U$, and any inverse $x$ of $s$ in $S$, since $s x \in U$, we have

$$
\begin{gathered}
(s x) \alpha_{s}=s, \\
(s x) \beta_{s}=(s x) \phi^{-1} \alpha_{s} \phi=(s x) \alpha_{s} \phi=s \phi=s^{\prime},
\end{gathered}
$$

and so $\alpha_{s} \neq \beta_{s}$.

We now take the direct sum of each of $\alpha$ and $\beta$ with the right regular representation of $S$, thus obtaining two monomorphisms from $S$ into a single semigroup, agreeing precisely on $U$. Thus the amalgam $(S, S ; U)$ is strongly embeddable in a [finite] semigroup [if $S$ is finite]. This completes the proof of the lemma.

REMARK 1. If $U$ is a subsemigroup of a finite semigroup $S$ and if $U$ is closed in $S$, then it is always the case that the amalgam $(S, S ; U)$ can be 
strongly embedded in a finite semigroup. To see this, we modify the proof of [9, Theorem VII.2.5] by taking the free abelian group of exponent 2, say, on $A$, instead of taking the free abelian group on $A$.

LEMMA 7. If $U$ is any full regular subsemigroup of regular semigroups $S$ and $T$ then the amalgam $(S, T ; U)$ is weakly embeddable in a semigroup. If $S$ and $T$ are finite then the amalgam can be weakly embedded in a finite semigroup.

Proof. First we note that for any $x \in S, u \in U$, if $(x, x u) \in \rho(S)_{u}$ then for any inverse $x^{\prime}$ of $x$ in $S$, since $x \Re x u$ in $S$ we have $x^{\prime} x \Re x^{\prime} x u$ in $S$ and hence in $U$ (Result 1), so for some element $v \in U$ (rather than $U^{1}$, since $U$ is regular), $x^{\prime} x=x^{\prime} x u v$ whence $x=(x u) v$. This shows that $U$ acts transitively on each $U$-orbit of $\rho(S) \mid U$.

We define two $U$-orbits $O$ and $O^{\prime}$ of $\rho(S) \mid U$ to be $\mathcal{L}$-equivalent if there are

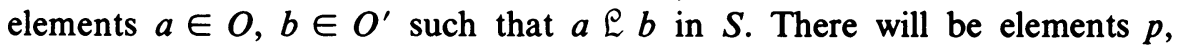
$q \in S^{1}$ such that $a=p b, b=q a$ and then routine calculations show that the maps $O \rightarrow O^{\prime}, x \mapsto q x$, and $O^{\prime} \rightarrow O, y \mapsto p y$ are mutually inverse, $\mathcal{L}$-class preserving isomorphisms between $U$-orbits of $\rho(S) \mid U$. Also, for any element $x \in O$ and any inverse $x^{\prime}$ of $x$ in $S$, the $U$-orbit of $\rho(S) \mid U$ containing $x^{\prime} x, O^{\prime \prime}$ say, is $\mathcal{L}$-equivalent to $O$ and is also a $U$-orbit of $\rho(U)$ (note that $U$ has the same action on $O^{\prime \prime}$ under $\rho(U)$ and $\left.\rho(S) \mid U\right)$. Thus the isomorphism types of $U$-orbits of $\rho(S) \mid U$ are precisely those of $\rho(U)$, and hence precisely those of $\rho(T) \mid U$. As in the proof of Theorem 1, from $\alpha=\rho(S)$ and $\beta=\rho(T)$ construct representations $\alpha^{\prime \prime}: S \rightarrow \mathcal{P} \mathcal{T}\left(Z_{2}^{\prime}\right)$ and $\beta: T \rightarrow \mathcal{P} \mathcal{T}\left(Z_{2}^{\prime}\right)$ agreeing on $U$. Dually, from $\lambda(S)$ and $\lambda(T)$ construct homomorphisms $\bar{\alpha}^{\prime \prime}$ and $\bar{\beta}$ agreeing on $U$, imitating each detail in the dual situation. Then $\left(\alpha^{\prime \prime}, \bar{\alpha}^{\prime \prime}\right)$ and $(\beta, \bar{\beta})$, defined as usual, are monomorphisms agreeing on $U$. Hence the amalgam $(S, T ; U)$ is weakly embeddable. It remains to show that finiteness can be preserved, so we assume now that $S$ and $T$ are finite.

Let us first note that the $S$-orbits of $\rho(S)$ are just the $R$-classes of $S$, on

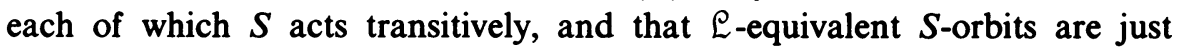
$\Re$-classes contained in the same $\mathscr{D}$-class of $S$ (take the case where $U$ is all of $S)$.

Let $S_{1}, \ldots, S_{m}$, and $T_{1}, \ldots, T_{n}$, and $U_{1}, \ldots, U_{p}$ say, be $\mathcal{R}$-classes from $S, T$ and $U$, with precisely one $\Re$-class chosen from each $\mathscr{D}$-class of $S, T$ and $U$, respectively; denote the cardinality of a $\mathcal{H}$-class of $S, T$ and $U$ in these $\Re$-classes by $\delta_{1}, \ldots, \delta_{m}$, and $\xi_{1}, \ldots, \xi_{n}$, and $\eta_{1}, \ldots, \eta_{p}$, respectively.

Now $S_{1}$ is an $S$-orbit of $\rho(S)$ and so contains complete $U$-orbits of $\rho(S) \mid U$, and each such is $\mathcal{L}$-equivalent to one of $U_{1}, \ldots, U_{p}$, each of these being the same $U$-orbit of $\rho(S) \mid U$ as of $\rho(U)$. Further, if $S_{1}$ contains a $U$-orbit $\mathcal{L}$-equivalent to $U_{i}$ say, then $S_{1}$ contains precisely $\delta_{1} / \eta_{i} U$-orbits $\mathcal{L}$-equivalent to $U_{i}$ (each a copy of $U_{i}$ ), which we prove as follows. Suppose $U_{i}$ meets exactly $q$, say, $\mathfrak{L}$-classes of $U$ (equivalently, of $S$, by Result 1 ). Then 
$\left|U_{i}\right|=q \eta_{i}$. Let $V$ be the set of elements of $S_{1}$ which are $\mathcal{L}$-related in $S$ to elements of $U_{i}$. Then $V$ is a disjoint union of $q \mathcal{H}$-classes of $S_{1}$ so $|V|=q \delta_{1}$. Also $V$ consists of the disjoint union of those $U$-orbits of $\rho(S) \mid U$ which are $\mathcal{L}$-equivalent to $U_{i}$ and contained in $S_{1}$, so the number of such $U$-orbits is

$$
|V| /\left|U_{i}\right|=q \delta_{1} / q \eta_{i}=\delta_{1} / \eta_{i}
$$

as required. Similar remarks apply to $S_{2}, \ldots, S_{m}, T_{1}, \ldots, T_{n}$.

Put $N=\delta_{1} \delta_{2} \cdots \delta_{m} \xi_{1} \xi_{2} \cdots \xi_{n}$. Take the sum of $N / \delta_{i}$ copies of $\rho(S) \| S_{i}$, $i=1,2, \ldots, m$, and take the sum of all these sums, denoting the final sum by $\alpha$. Since $\alpha$ contains each $S$-orbit type of $\rho(S)$ we have $\alpha \circ \alpha^{-1}=$ $\rho(S) \circ \rho(S)^{-1}$. Take the sum of $N / \xi_{j}$ copies of $\rho(T) \| T_{j}, j=1,2, \ldots, n$, and take the sum of all these sums, denoting the final sum by $\beta$.

We consider $\alpha \mid U$ and $\beta \mid U$. Take any $U_{i}$ and for convenience suppose $S_{1}$ and $T_{1}$ contain $U$-orbits of $\rho(S) \mid U$ and $\rho(T) \mid U$, respectively, $\mathcal{L}$-equivalent to $U_{i}$. Then the $N / \delta_{1}$ copies of $\rho(S) \| S_{1}$ contain $\left(N / \delta_{1}\right)\left(\delta_{1} / \eta_{i}\right)=N / \eta_{i}$ copies of $U_{i}$ and the $N / \xi_{1}$ copies of $\rho(T) \| T_{1}$ contain $\left(N / \xi_{1}\right)\left(\xi_{1} / \eta_{i}\right)=N / \eta_{i}$ copies of $U_{i}$.

We now see that $\alpha \mid U$ is the sum of $N / \eta_{1}$ copies of $U_{1}, N / \eta_{2}$ copies of $U_{2}, \ldots$, and $N / \eta_{p}$ copies of $U_{p}$, and so also is $\beta \mid U$. Hence $\alpha \mid U$ and $\beta \mid U$ are equivalent so there is a one-to-one function $\phi$ say, such that $\phi^{-1} \alpha_{u} \phi=\beta_{u}$ for all $u \in U$. Defining $\alpha_{s}^{\prime}=\phi^{-1} \alpha_{s} \phi$ for all $s \in S$ gives us representations $\alpha^{\prime}$ of $S$ and $\beta$ of $T$ agreeing on $U$. From $\lambda(S)$ and $\lambda(T)$ let us produce $\bar{\alpha}^{\prime}$ and $\bar{\beta}$, just as from $\rho(S)$ and $\rho(T)$ we produced $\alpha^{\prime}$ and $\beta$, imitating each detail above in the dual situation. Then $\left(\bar{\alpha}^{\prime}, \alpha^{\prime}\right)$, defined as usual, is faithful on $S$, as is $(\bar{\beta}, \beta)$ on $T$, and the two monomorphisms agree on $U$, giving that the amalgam $(S, T ; U)$ can be weakly embedded in a finite semigroup.

THEOREM 8. Let $\left(S_{i}, i \in I ; U\right)$ be any amalgam of semigroups, all regular, and such that $U$ contains all the idempotents of $S_{i}$, for all $i \in I$. Then the amalgam is strongly embeddable. If $I$ and each $S_{i}$ are finite then the amalgam is strongly embeddable in a finite semigroup. For any semigroup $W$ such that $S_{i} \leqslant W$ for all $i \in I$ and such that $W$ is generated by $\cup_{i \in I} S_{i}, W$ is regular and $E(W)=E(U)$.

Proof. We deal first with the case $I=\{1,2\}$ say. Put $S=S_{1}, T=S_{2}$. Then from Lemma $7,(S, T ; U)$ can be weakly embedded in a semigroup $V$ say, by monomorphisms $\phi: S \rightarrow V, \psi: T \rightarrow V$, agreeing on $U$. Without loss of generality we can assume $V$ is generated by $(S \phi) \cup(T \psi)$. Then from Result 2 , $V$ is regular and $E(U \phi)=E(V)$. By Lemma 6 , the amalgam $(V, V ; U \phi)$ can be strongly embedded in a semigroup $W$ by monomorphisms $\phi^{\prime}: V \rightarrow W, \psi^{\prime}$ : $V \rightarrow W$ agreeing precisely on $U \phi$. Then the monomorphisms $\phi \phi^{\prime}: S \rightarrow W$ and $\psi \psi^{\prime}: T \rightarrow W$ agree precisely on $U$. Hence $(S, T ; U)$ is strongly embeddable. Further, at each step finiteness can be preserved so $W$ can be taken to be 
finite if $S$ and $T$ are finite. The fact that $W$ is regular and that $E\left(U \phi \phi^{\prime}\right)=$ $E(W)$ enables us to obtain the strong embedding for an arbitrary index set $I$ by transfinite induction, embedding into a finite semigroup when $I$ and each $S_{i}$ are finite. The last statement of the theorem follows from Result 2.

Corollary 9. An amalgam $\left(S_{i}, i \in I ; U\right)$ of orthodox semigroups such that $U$ is full in each $S_{i}$ is strongly embeddable in an orthodox semigroup.

Remark 2. This latter result preceded and led the author to Theorem 8. The original proof quite easily deduced Corollary 9 from the strong amalgamation property for inverse semigroups and the author's construction [6, Theorem 1] of orthodox semigroups in terms of bands and inverse semigroups.

4. Unions of groups. The following theorem is well known for inverse semigroups. For orthodox semigroups, it has been announced as an unpublished result of the present author by Clifford in [2], where it played a major rôle in giving a further structure theorem for orthodox unions of groups. We shall obtain from it a result concerning amalgamation of unions of groups.

THEOREM 10. Let $E$ be the set of all idempotents of any regular semigroup $S$.

(i) There is a full subsemigroup $U$ of $S$ which is a union of groups if and only if $\langle E\rangle$, the subsemigroup generated by $E$, is a union of groups.

(ii) In that case, among the full subsemigroups which are unions of groups there is a maximum member, namely

$$
\begin{aligned}
& E^{U}=\left\{a \in S: \text { for some [all] } a^{\prime} \in V(a), a^{\prime} x a g x\right. \\
& \left.\qquad \text { in }\langle E\rangle \text { for all } x \in E \text { with } x \leqslant a a^{\prime}\right\} .
\end{aligned}
$$

Proof. (i) The subsemigroup generated by the idempotents of a regular semigroup is regular (Fitz-Gerald [3]) and then from Result 1 and its dual it is easily seen that the subsemigroup generated by the idempotents of a union of groups is also a union of groups.

(ii) For the remainder of the proof $V(a)$ will denote the set of inverses in $S$ of an element $a \in S$, and $g$ will denote Green's relation $g$ on $\langle E\rangle$; of course $g$ is a congruence on $\langle E\rangle$ and $\langle E\rangle / \mathcal{g}$ is a semilattice. Let

$$
\begin{aligned}
& T_{1}=\left\{a \in S: \text { for some } a^{\prime} \in V(a), a^{\prime} x a g x \text { in }\langle E\rangle\right. \\
& \text { for all } \left.x \in E \text { with } x \leqslant a a^{\prime}\right\}, \\
& T_{2}=\left\{a \in S: \text { for all } a^{\prime} \in V(a), a^{\prime} x a g \text { in }\langle E\rangle\right. \\
& \text { for all } \left.x \in E \text { with } x \leqslant a a^{\prime}\right\} ;
\end{aligned}
$$

clearly $T_{2} \subseteq T_{1}$.

Take any elements $a, b \in T_{1}$ and any elements $a^{\prime} \in V(a), b^{\prime} \in V(b)$ satisfying the condition of membership of $a, b$ in $T_{1}$. 
Take any $y \in V\left(a^{\prime} a b b^{\prime}\right)$ and put $z=b^{\prime} y a^{\prime}$. Then routine checking shows that $z \in V(a b)$ and $b z a \in E$. Further

$$
b z a=(b z a)\left(a^{\prime} a\right) \mathcal{g}\left(a^{\prime} a\right)(b z a)=a^{\prime}(a b z) a g a b z
$$

since $a b z \leqslant a a^{\prime}$.

Take any $x \in E$ with $x \leqslant a b z\left(\leqslant a a^{\prime}\right)$. Then $a^{\prime} x a \in E$, and $a^{\prime} x a g x$. Further

$$
z x a b=b^{\prime}\left(b z x a b b^{\prime}\right) b=b^{\prime} w b g w
$$

since $w=b z x a b b^{\prime}$ say, is an idempotent (routine to check from $x<a b z$ ) and $w \leqslant b b^{\prime}$. Now $w=(b z a)\left(a^{\prime} x a\right)\left(b b^{\prime}\right)$, so in the semilattice $\langle E\rangle / g$ we have

$$
\begin{aligned}
J_{z x a b} & =J_{w}=J_{b z a} J_{a^{\prime} x a} J_{b b^{\prime}}=J_{b b^{\prime}} J_{b z a} J_{a^{\prime} x a} \\
& =J_{b z a} J_{a^{\prime} x a}=J_{a b z} J_{x}=J_{a b z x}=J_{x} .
\end{aligned}
$$

Hence $z x a b g x$ and $a b \in T_{1}$ giving that $T_{1}$ is a subsemigroup of $S$. Further, for any $y \in E$ with $y \leqslant a^{\prime} a$ we have $y=a^{\prime} a y a^{\prime} a=a^{\prime}\left(a y a^{\prime}\right) a g$ ay $a^{\prime}$ since $a y a^{\prime} \in E$ and $a y a^{\prime} \leqslant a a^{\prime}$, giving that $a^{\prime} \in T_{1}$ and so $T_{1}$ is regular.

Also $a^{\prime} a=a^{\prime}\left(a a^{\prime}\right) a g a a^{\prime}$ so there is an element $c \in\langle E\rangle$ such that $a^{\prime} a \mathcal{L} c \Re a a^{\prime}$ in $\langle E\rangle(\mathcal{G}=\mathscr{D}$ on $\langle E\rangle)$ and hence also in $S$. But $a^{\prime} a \mathcal{L}$ $a \Re a a^{\prime}$ so $a \mathcal{H} c$ in $S$. Since $\langle E\rangle$ is a union of groups, $c \mathcal{H} e$ in $\langle E\rangle$ for some $e \in E$, whence $a \mathcal{H} e$ in $S$, and thus in $T_{1}$ by Result 1 and its dual. Thus $T_{1}$ is a union of groups. Clearly $E \subseteq T_{1}$.

Now let $T$ be any full subsemigroup of $S$ which is a union of groups and take any $a \in T$ and any inverse $a^{\prime}$ of $a$ in $S$. Then $a^{\prime} \in T$ by Result 1 and its dual and [1, Theorem 2.18]. Take any $x \in E$ with $x \leqslant a a^{\prime}$. In the semilattice $T / \mathscr{D}$ we have

$$
D_{a^{\prime} x a}=D_{a^{\prime}} D_{x} D_{a}=D_{x} D_{a} D_{a^{\prime}}=D_{x a a^{\prime}}=D_{x},
$$

i.e. $a^{\prime} x a \mathscr{D} x$ in $T$. One easily obtains that $a^{\prime} x a \mathscr{D} x$ in $\langle E\rangle$, i.e. $a^{\prime} x a \mathscr{G} x$, giving that $a \in T_{2}$. Hence $T \subseteq T_{2} \subseteq T_{1}$, giving $T_{1} \subseteq T_{2}$ and the required result.

REMARK 3. The above theorem and manipulative proof were both derived from the author's fundamental representation $(\rho, \lambda): S \rightarrow T_{\langle E\rangle}$ of any regular semigroup $S$ with set of idempotents $E$ in $T_{\langle E\rangle}$ [5, Theorem 7], a generalization to all regular semigroups of the Munn semigroup $T_{E}$ for inverse semigroups. An outline of this conceptual proof is as follows. Construct $T_{\langle E\rangle}$ and $(\rho, \lambda): S \rightarrow T_{\langle E\rangle}$. Using the formula $\phi(\alpha) \phi(\beta)=\phi(\delta)$ of $[5$, p. 11] we easily characterize $E^{U}$ in $T_{\langle E\rangle}$ as

$$
\begin{aligned}
& \left\{\phi(\alpha) \text { : for some } e, f \in E, \alpha \in T_{e_{f}}\right. \text { and } \\
& \qquad x \alpha g \text { in }\langle E\rangle \text { for all } x \in E \text { with } x \leqslant e\} .
\end{aligned}
$$

Then $E^{U}$ in $S$ is found as the set of elements of $S$ mapping under $(\rho, \lambda)$ into the $E^{U}$ of $T_{\langle E\rangle}$. 
For $S$ an orthodox semigroup another simple conceptual proof takes the back image in $S$ of $E^{U}$, the centralizer of the set of idempotents, in the maximum inverse semigroup homomorphic image of $S$.

REMARK 4. Note that by [5, Theorem 3], $\langle E\rangle$ is a union of groups if and only if for all $e, f, g \in E$ such that $e \mathcal{L} f \Re g$ in $S$ there exists $h \in E$ such that $e \Re h \mathcal{L}$.

Corollary 11. Let $E$ be any set of idempotents of a semigroup $S$.

(i) There is a subsemigroup $U$ of $S$ which is a union of groups having $E$ as its set of all idempotents if and only if $\langle E\rangle$, the subsemigroup generated by $E$, is such a semigroup.

(ii) If $\langle E\rangle$ is a union of groups having $E$ as its set of all idempotents then there is a maximum such subsemigroup of $S$, namely

$$
\begin{aligned}
& E^{U}=\left\{a \in S: \text { for some } a^{\prime} \in V(a), a^{\prime} a, a a^{\prime}, a^{\prime} x a, a y a^{\prime} \in E\right. \\
& \left.\quad \text { and } a^{\prime} x a g \text { in }\langle E\rangle \text { for all } x, y \in E \text { with } x \leqslant a a^{\prime}, y<a^{\prime} a\right\} .
\end{aligned}
$$

Proof. This follows from Theorem 10 and [5, Theorem 14].

Corollary 12. An amalgam $\left(S_{i}, i \in I ; U\right)$ of [orthodox, inverse] semigroups which are unions of groups such that $U$ is full in each $S_{i}$ can be strongly embedded in an [orthodox, inverse] union of groups, with finiteness being preserved.

Proof. This follows from Theorems 8 and 10.

ACKNOWLEDGEMENT. I thank my friend and colleague Chris Ash for the main idea of this paper, that of taking the sum of $\lambda$ copies of two representations with the same $U$-orbit types.

\section{REFERENCES}

1. A. H. Clifford and G. B. Preston, The algebraic theory of semigroups, Vols. 1, 2, Math. Surveys, no. 7, Amer. Math. Soc., Providence, R.I., 1961, 1967.

2. A. H. Clifford, A structure theorem for orthogroups, J. Pure Appl. Algebra 8 (1976), 23-50.

3. D. G. Fitz-Gerald, On inverses of products of idempotents in regular semigroups, J. Austral. Math. Soc. 13 (1972), 335-337.

4. T. E. Hall, On orthodox semigroups and uniform and anti-uniform bands, J. Algebra 16 (1970), 204-217.

5. __ On regular semigroups, J. Algebra 24 (1973), 1-24.

6. __ Orthodox semigroups, Pacific J. Math. 39 (1971), 677-686.

7. __ Free products with amalgamation of inverse semigroups, J. Algebra 34 (1975), $375-385$.

8. __ Representation extension and amalgamation for semigroups, Quart J. Math. Oxford (to appear).

9. J. M. Howie, An introduction to semigroup theory, London Math. Soc. Monographs no. 7, Academic Press, London and New York, 1976.

10. Gerard Lallement, Structure theorems for regular semigroups, Semigroup Forum 4 (1972), 95-123. 
11. G. B. Preston, Matrix representations of semigroups, Quart. J. Math. Oxford Ser. (2) 9 (1958), 169-176.

12. Inverse semigroups: some open questions, Proc. Sympos.Inverse Semigroups and Their Generalizations, Northern Illinois Univ., De Kalb, Ill., 1973, pp. 122-139.

13. __ Representations of inverse semigroups by one-to-one partial transformations of a set, Semigroup Forum 6 (1973), 240-245; Addendum, Semigroup Forum 8 (1974), 277.

14. - Free products amalgamating unitary subsemigroups, Bull. Austral. Math. Soc. 15 (1976), 117-124.

15. ___ Free products amalgamating almost unitary subsemigroups (to be submitted).

Department of Mathematics, Monash University, Clayton, 3168, Australia 